El ect ri cal - resi st i vi ty and I ow t emper at ure speci fi c- heat measur ement s of si ngl e crystal s of thi ospi nel CuV2S4

\begin{tabular}{|l|l|}
\hline 著者 & $\begin{array}{l}\text { HAG NO T., SEK Y., TAKAYANAG S., WADA } \\
\text { N., NAGATA Shoi chi }\end{array}$ \\
\hline $\begin{array}{l}\text { j our nal or } \\
\text { publ i cat i on t i t l e }\end{array}$ & $\begin{array}{l}\text { Physi cal r evi ew. Thi } r \text { d ser i es. B, Condensed } \\
\text { mat t er }\end{array}$ \\
\hline vol une & 49 \\
\hline number & 10 \\
\hline page $r$ ange & $6822-6828$ \\
\hline year & 1994 03-01 \\
\hline URL & ht t p: //hdl . handl e. net /10258/256 \\
\hline
\end{tabular}




\title{
Electrical-resistivity and low-temperature specific-heat measurements of single crystals of thiospinel $\mathrm{CuV}_{2} \mathrm{~S}_{4}$
}

\author{
Takatsugu Hagino and Yoshitaka Seki \\ Department of Materials Science and Engineering, Muroran Institute of Technology, Mizumoto-cho 27-1, Muroran 050, Japan \\ Shigeru Takayanagi \\ Physics Department, Sapporo Campus, Hokkaido-Kyouiku University, Sapporo 002, Japan \\ Nobuo Wada \\ Department of Physics, College of Arts and Science, University of Tokyo, Tokyo 153, Japan \\ Shoichi Nagata \\ Department of Materials Science and Engineering, Muroran Institute of Technology, Mizumoto-cho 27-1, Muroran 050, Japan
}

(Received 4 October 1993; revised manuscript received 29 November 1993)

\begin{abstract}
Zero-field and magnetic-field low-temperature specific-heat measurements have been carried out on single crystals of $\mathrm{CuV}_{2} \mathrm{~S}_{4}$. The electronic-specific-heat coefficient (in zero field) is found to be 62 $\mathrm{mJ} / \mathrm{mol} \mathrm{K}^{2}$ and the Debye temperature to be $205 \mathrm{~K}$. A superconducting transition at $4.0 \mathrm{~K}$ has been reported in powder samples contaminated by an estimated 5 to $10 \%$ sulvanite $\mathrm{Cu}_{3} \mathrm{VS}_{4}$. Present experiments of the electrical resistivity and the specific heat, however, verify that high-quality single crystals do not exhibit a superconducting transition above $60 \mathrm{mK}$. Below $0.2 \mathrm{~K}$, nuclear specific heat is observed. Applied magnetic fields increase the magnitude of the specific heat due to the nuclear Zeeman effect of $\mathrm{V}$ and $\mathrm{Cu}$ nuclei and the electric quadrupole interaction of $\mathrm{Cu}$ nuclei. The electric-field gradient at $\mathrm{Cu}$ nuclei is estimated to be eq $=1.74 \times 10^{22} \mathrm{~V} / \mathrm{m}^{2}$. Detailed analysis of the nuclear specific heat below $1.0 \mathrm{~K}$ is presented. A comparison of the theoretical analysis with the experimental result is given and are in excellent agreement.
\end{abstract}

\section{INTRODUCTION}

The ternary thiospinel $\mathrm{CuV}_{2} \mathrm{~S}_{4}$ has the cubic spinel structure at room temperature which has a lattice constant of $a=9.808 \AA$, as shown in Fig. 1. $\mathrm{CuV}_{2} \mathrm{~S}_{4}$ has the normal spinel structure where $\mathrm{Cu}$ ions occupy the $A$ (tetrahedral) sites and $\mathrm{V}$ ions occupy the $B$ (octahedral) sites. This compound exhibits phase transitions associated with charge-density waves (CDW's) at 55, 75, and 90 $\mathrm{K}$. These CDW transitions are rare in three-dimensional cubic structures, which is why intensive studies have been made. ${ }^{1-4}$ We will not focus in this paper on the subject of the CDW.

On the other hand, it has been reported that $\mathrm{CuV}_{2} \mathrm{~S}_{4}$ reveals superconductivity with a transition temperature in the range 4.45-3.95 $\mathrm{K}$ measured on powder samples contaminated with sulvanite $\mathrm{Cu}_{3} \mathrm{VS}_{4}{ }^{5}$ In this paper, we will make clear whether a pure $\mathrm{CuV}_{2} \mathrm{~S}_{4}$ single crystal exhibits superconductivity around $4.0 \mathrm{~K}$ or not. Consequently, contrary to the earlier results, we confirm that the high-quality single crystals do not show any superconducting transition in a temperature region above 60 $\mathrm{mK}$. Thermal properties in single crystals of $\mathrm{CuV}_{2} \mathrm{~S}_{4}$ have been studied with special emphasis on the lowtemperature range. The electric-field gradient at $\mathrm{Cu}$ nuclei is discussed in connection with a Jahn-Teller distortion (local distortion at $A$ sites in the crystal). The contribution to the specific heat due to the nuclear Zeeman effect of $\mathrm{Cu}$ and $\mathrm{V}$ in magnetic fields is observed clearly. These magnitudes of the specific heat in the Schottkytype anomaly are calculated theoretically and comparison with experimental results leads to excellent agreement. A brief report has been given earlier; ${ }^{6}$ here, more detailed results will be presented.

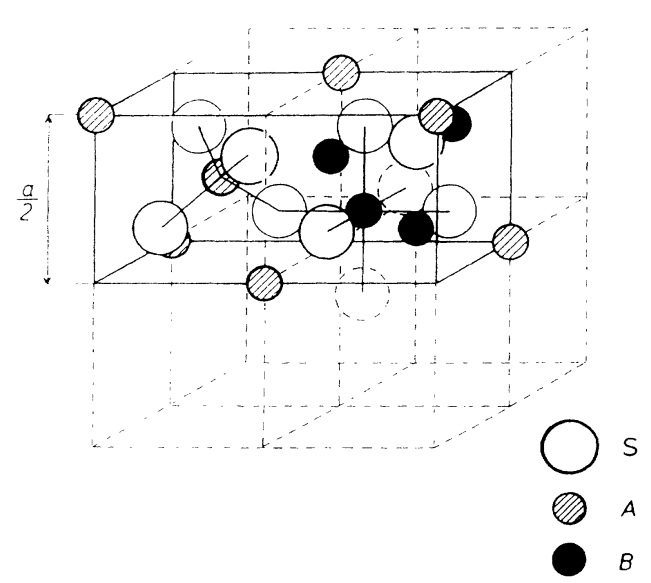

FIG. 1. Unit cell of spinel structure. The position of the ions in only two octants is shown. Large circles indicate sulfur ions, small hatched circles are copper ions at tetrahedral $A$ sites, and small solid circles are vanadium ions at octahedral $B$ sites. 


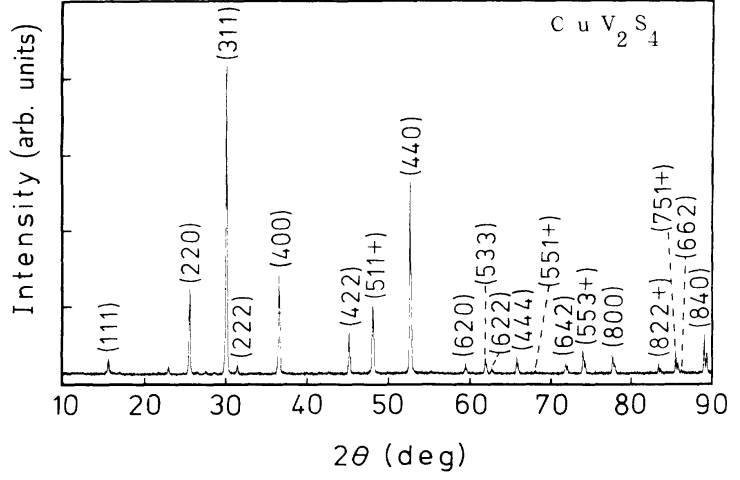

FIG. 2. X-ray-diffraction pattern of $\mathrm{CuV}_{2} \mathrm{~S}_{4}$ at $300 \mathrm{~K}$.

\section{EXPERIMENTAL METHODS}

\section{A. Sample preparation}

The single crystals were synthesized in a quartz ampoule by $\mathrm{TeCl}_{4}$ vapor transport at growth temperatures from 830 to $720^{\circ} \mathrm{C}$ in a two-zone furnace. ${ }^{7}$ The crystals grow as octahedrons with a maximum size of about $3 \times 3 \times 3 \mathrm{~mm}^{3}$. The $x$-ray-diffraction pattern in powder specimens is shown in Fig. 2. The pure spinel phase is obtained. Back-reflected x-ray Laue diffraction reveals (Fig. 3) that the eight equivalent $\langle 111\rangle$ directions are aligned normal to the crystal faces. X-ray diffraction also confirms the pure spinel phase in powder specimens.

\section{B. Electrical resistivity measurements}

The resistivity was measured by a standard four-probe method. Silver paste was used to fabricate the electrodes. The temperature dependence of the resistivity was measured over the region $1.5-290 \mathrm{~K}$ for the single crystal.

\section{Specific-heat measurements}

The specific-heat measurements were performed in the temperature range over $60 \mathrm{mK}-20 \mathrm{~K}$ using a ${ }^{3} \mathrm{He} /{ }^{4} \mathrm{He}$

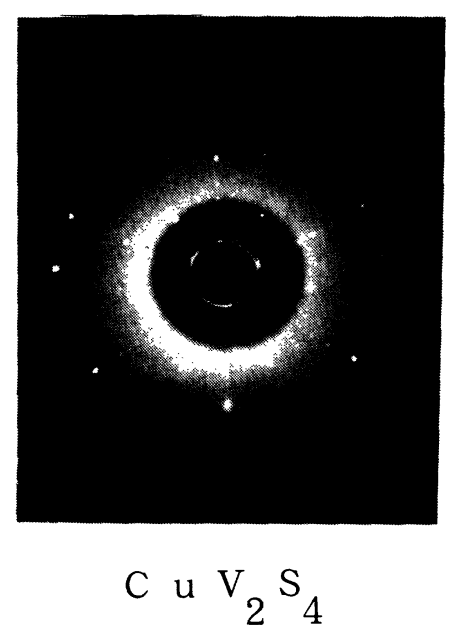

FIG. 3. Laue photograph for $\mathrm{CuV}_{2} \mathrm{~S}_{4}$ taken by back reflection from a face of octahedral-shaped single crystal. dilution refrigerator and $a{ }^{3} \mathrm{He}$ cryostat in both the absence and presence of an applied magnetic field of $5.0 \mathrm{~T}$ in continuous operation. Several single crystals with a total mass of $\sim 1.0 \mathrm{~g}$ were used for the measurements.

\section{RESULTS AND DISCUSSION}

\section{A. Electrical resistivity}

Figure 4 shows the resistivity $\rho$ as a function of temperature. The temperature dependence has almost the same shape as reported by Le Nagard et al. ${ }^{2}$ With decreasing temperature from $290 \mathrm{~K}, \rho$ decreases and begins increasing gradually at $130 \mathrm{~K}$ and then decreases again with a maximum value at $50 \mathrm{~K}$. We could not observe a pronounced hysteresis around $55 \mathrm{~K}$; i.e., a dependence on the direction in which the sample is swept in cooling or heating processes. An argument for the anomaly due to CDW formation between 55 and $90 \mathrm{~K}$ is not given here because the discussion has already been given. ${ }^{1-4}$ The inset in Fig. 4 shows the resistivity below $10 \mathrm{~K}$. It should be noted that no anomaly in the resistivity associated with the superconducting transition has been found around $4 \mathrm{~K}$. On the contrary, $\rho$ remains essentially constant between 1.5 and $10 \mathrm{~K}$.

\section{B. Specific-heat results: $C$ vs $T$ curves}

The temperature dependences of the specific heat of single crystals of $\mathrm{CuV}_{2} \mathrm{~S}_{4}$ in the magnetic fields are shown in Figs. 5 and 6. Above $1.0 \mathrm{~K}$, the specific heat is unchanged in a magnetic field of $5.0 \mathrm{~T}$ within our experimental errors. Below $0.2 \mathrm{~K}$, the specific heat exhibits anomalous behavior and depends strongly on the external magnetic fields, which will be discussed below.

\section{Debye temperature and electronic specific heat}

The ratio of the specific heat to temperature, $C / T$ vs $T^{2}$ gives a fairly good straight line below $7.0 \mathrm{~K}$. The specific heat is well fitted by $C=\gamma T+A T^{3}$. The value of

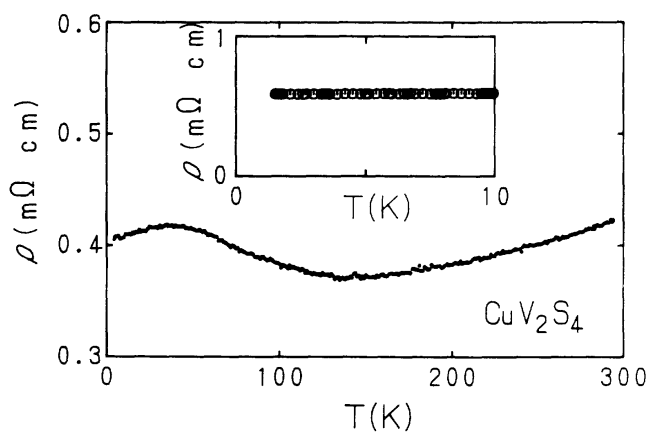

FIG. 4. Temperature dependence of the resistivity of single crystal $\mathrm{CuV}_{2} \mathrm{~S}_{4}$. The anomaly is associated with the chargedensity-wave formation between 55 and $90 \mathrm{~K}$. Inset: Resistivity of the single crystal of $\mathrm{CuV}_{2} \mathrm{~S}_{4}$ as a function of temperature. The difference in the magnitude of $\rho$ originates from errors in measurement of the length of electrodes in the small crystal. The sample is the same as specified in these figures. 


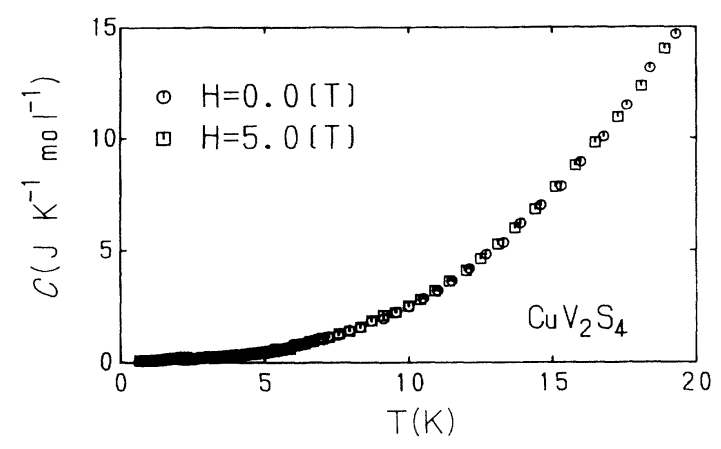

FIG. 5. The specific heat of $\mathrm{CuV}_{2} \mathrm{~S}_{4}$ in the absence of applied magnetic field $(O)$ over the temperature region of $0.7-20 \mathrm{~K}$. The specific heat of $\mathrm{CuV}_{2} \mathrm{~S}_{4}$ at a magnetic field of $5.0 \mathrm{~T}$ ( $\square$ ) over the temperature region of $0.7-20 \mathrm{~K}$.

$\gamma=50 \mathrm{~mJ} / \mathrm{K}^{2} \mathrm{~mol}$ is extracted from the intercept of the straight line.

Below $1.0 \mathrm{~K}$, the contribution of the lattice vibration to the specific heat is negligibly small, then the $C / T$ vs $T$ plot is given in Fig. 7, where the value of $\gamma=62$ $\mathrm{mJ} / \mathrm{K}^{2} \mathrm{~mol}$ is extracted from the flat part. The magnitude of $\gamma$ is rather large, which would give a high density of states at the Fermi surface.

From the value of $A=1.59 \mathrm{~mJ} / \mathrm{K}^{4}$ mol-f.u., we estimate the Debye temperature $\Theta$ using the relation

$$
A=\frac{12 \pi^{4} r N_{0} k_{B}}{5 \Theta^{3}} .
$$

Here $r$ is the number of atoms per formula unit, $N_{0}$ is Avogadro's number, and $k_{B}$ is Boltzmann's constant. Equation (1) gives $\Theta=205 \mathrm{~K}$ with $r=7$ as the formula unit. The Debye temperature of $\mathrm{CuV}_{2} \mathrm{~S}_{4}$ is extremely small in comparison with the value of an oxide spinel compound, such as $\mathrm{LiTi}_{2} \mathrm{O}_{4},{ }^{8}$ which has the value of $A=0.043 \mathrm{~mJ} / \mathrm{K}^{4}$ mol-f.u., so that $\Theta=685 \mathrm{~K}$.

An origin of the low value in the Debye temperature of $\mathrm{CuV}_{2} \mathrm{~S}_{4}$ might be attributed to the rattling cations with correspondingly large spaces among the sulfur anions having large ionic radius. In sulfides such as $\mathrm{CuV}_{2} \mathrm{~S}_{4}$, the cations have large room for moving in the sulfur anion network. Consequently, the lattice vibration can easily be excited in the crystal. The difference in Debye temperatures between the oxides and sulfides could come from this rattling cation's effect in the sulfides.

A summary of the electronic and lattice specific heats is given in Table I for various temperature regions of the data fitting.

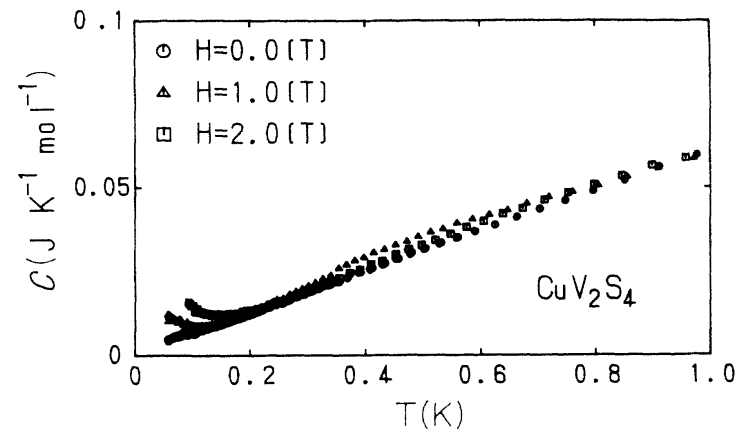

FIG. 6. The specific heat of $\mathrm{CuV}_{2} \mathrm{~S}_{4}$ at three magnetic fields over the temperature region $60 \mathrm{mK}-1.0 \mathrm{~K}$.

\section{Nuclear specific heat: Jahn-Teller distortion and electric-field gradient at $\mathrm{Cu}$ nuclei}

Figures 7-9 show a low-temperature anomaly in the specific heat even in the absence of the applied magnetic field. Furthermore the magnitude of the specific heat increases when the intensity of the applied magnetic field goes up. If the anomaly originates from superconductivity, the specific heat must diminish gradually with increasing intensity of the magnetic field. Therefore the origin of this anomaly does not come from superconductivity. In this section, we first discuss the anomalous behavior of the specific heat in the absence of the applied magnetic field.

The nuclear quadrupole splitting of $\mathrm{Cu}$ can give rise to a zero-field anomaly in the specific heat at low temperatures. The copper atom has a nuclear spin $I=\frac{3}{2}$, then, even in the case of absence of applied magnetic field, a nuclear quadrupole splitting arises when an electric-field gradient exists at the $\mathrm{Cu}$ nuclei. Copper exists in two isotopes ${ }^{63} \mathrm{Cu}(69.09 \%)$ and ${ }^{65} \mathrm{Cu}(30.91 \%)$, each of which has a nuclear spin $I=\frac{3}{2}$. The copper atom is located in the $A$ site with tetrahedral crystalline symmetry $T_{d}$, which has cubic symmetry in the normal spinel structure. In the cubic symmetry, the electric-field gradient is zero; consequently the nuclear quadrupole splitting does not appear and we cannot expect a specific-heat anomaly due to the quadrupolar moment of $\mathrm{Cu}$ nuclei. In spinel-type substances, the electronic orbital of $\mathrm{Cu}^{2+}$ at $A$ sites in $T_{d}$ symmetry is triply degenerated when the crystal is undistorted and is split into lower symmetry when the crystal is distorted. $^{9}$ This Jahn-Teller effect (local distortion at $A$ sites) can lead to an interaction between the electricfield gradient and the electric quadrupole of $\mathrm{Cu}$ nuclei. $\mathrm{A}$ nuclear-spin Hamiltonian is a sum of quadrupolar and

TABLE I. The characteristics of the electronic and lattice specific heats in $\operatorname{CuV}_{2} \mathrm{~S}_{4}: C=\gamma T+A T^{3}$. Here $r$ is the number of atoms per formula unit used as a definition in Eq. (1) and $\Theta$ is the Debye temperature.

\begin{tabular}{lccccc}
\hline & $0.3 \mathrm{~K}<T<1.0 \mathrm{~K}$ & $2 \mathrm{~K}<T<7.0 \mathrm{~K}$ & \multicolumn{2}{c}{$2 \mathrm{~K}<T<20 \mathrm{~K}$} \\
\hline$\gamma\left(\mathrm{mJ} / \mathrm{K}^{2} \mathrm{~mol}\right)$ & 62 & 50 & \multicolumn{2}{c}{56.7} \\
$A\left(\mathrm{~mJ} / \mathrm{K}^{4} \mathrm{~mol}\right)$ & & 1.59 & 1 & 1.98 \\
$r$ & & 1 & & 7 & 7 \\
$\Theta(\mathrm{K})$ & & 107 & 205 & 99.4 & 190 \\
\hline \hline
\end{tabular}




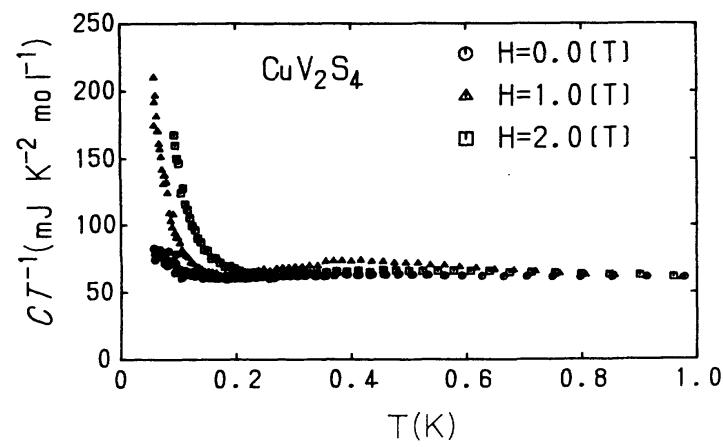

FIG. 7. The specific heat of $\mathrm{CuV}_{2} \mathrm{~S}_{4}$ plotted as $C / T$ vs $T$ from $60 \mathrm{mK}$ to $1.0 \mathrm{~K}$ at three magnetic fields.

Zeeman terms. With conventional notation, ${ }^{10}$

$$
\begin{aligned}
H= & \frac{e^{2} q Q}{4 I(2 I-1)}\left[3 I_{z}^{2}-I(I+1)+(1 / 2) \eta\left(I_{+}^{2}+I_{-}^{2}\right)\right] \\
& -\gamma_{N} \hbar I \cdot H,
\end{aligned}
$$

where $Q$ is the nuclear electric quadrupole moment, eq and $\eta$ are the magnitude and anisotropy parameter of the electric-field gradient (EFG), respectively, and $\gamma_{N}$ is the gyromagnetic ratio. We assume here an axially symmetric field gradient $(\eta=0)$. The energy is expressed as

$$
E(m)=\frac{3 e^{2} q Q}{4 I(2 I-1)} m^{2}, \quad I=3 / 2, I_{z}=m= \pm 3 / 2,
$$

and the energy difference $\Delta$ is given by

$$
\Delta=\frac{1}{2} e^{2} q Q \text {. }
$$

The present work is concerned primarily with evaluation of EFG at the Cu nuclei.

On the other hand, a Schottky-type specific heat is expressed as ${ }^{11}$

$$
\begin{aligned}
C_{v} & =R \frac{\Delta^{2}}{\left(k_{B} T\right)^{2}} \times \frac{\exp \left[\Delta /\left(k_{B} T\right)\right]}{\left\{1+\exp \left[\Delta /\left(k_{B} T\right)\right]\right\}^{2}}, \\
& =B / T^{2}, \quad B=R \Delta^{2} /\left(4 k_{B}^{2}\right), \quad k_{B} T \gg \Delta,
\end{aligned}
$$

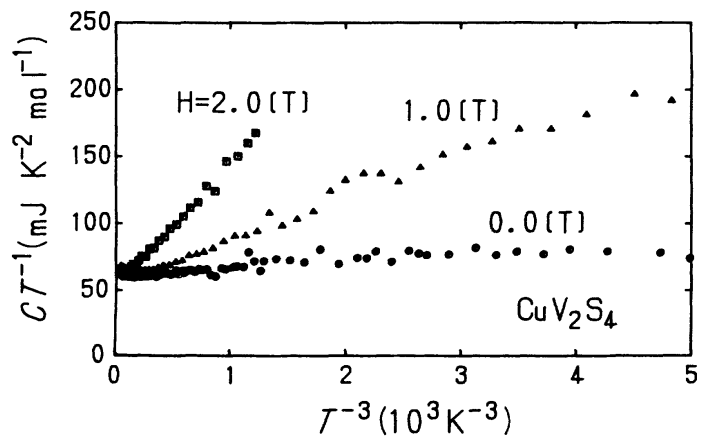

FIG. 8. The specific heat of $\mathrm{CuV}_{2} \mathrm{~S}_{4}$ plotted as $C / T$ vs $1 / T^{3}$ from $60 \mathrm{mK}$ to $1.0 \mathrm{~K}$ at three magnetic fields.

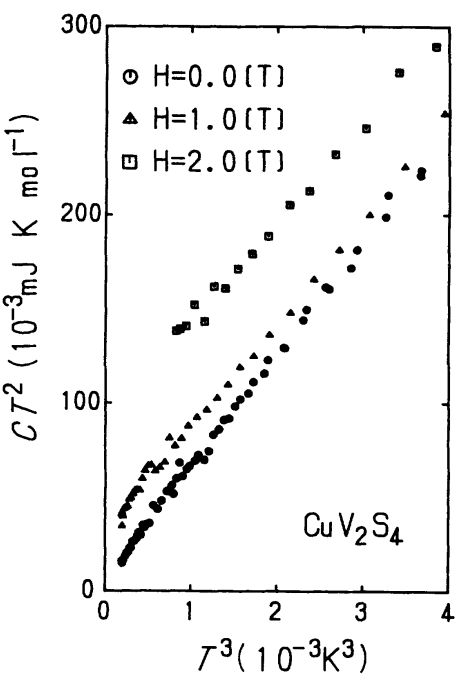

FIG. 9. The specific heat of $\mathrm{CuV}_{2} \mathrm{~S}_{4}$ plotted as $C T^{2}$ vs $T^{3}$ from 60 to $160 \mathrm{mK}$ at three magnetic fields.

where $R$ is the gas constant. The energy difference $\Delta$ is extracted from the value of $B$ which is the term linear in $1 / T^{2}$ in the specific heat for the high-temperature region $k_{B} T \gg \Delta$. The basic data in this work are summarized in Table II. The magnitude of the electronic-field gradient $e q$ is obtained:

$$
e q=1.74 \times 10^{22}\left[\mathrm{~V} / \mathrm{m}^{2}\right],
$$

which is indicated in Table III.

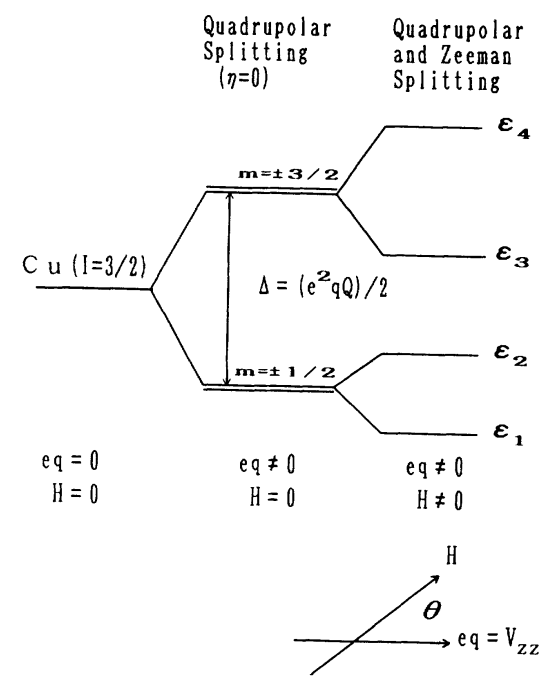

FIG. 10. The nuclear quadrupole splitting and the Zeeman splitting for the $\mathrm{Cu}$ nuclear spin $I=\frac{3}{2}$ in the absence and the presence of magnetic field. The axially symmetric field gradient $(\eta=0)$ is supposed when the Jahn-Teller distortion is caused. The energy levels are calculated when the principal axis in the electric-field gradient is inclined at an angle $\theta$ between this symmetric axis and the applied magnetic field. 
TABLE II. Numerical values for $\mathrm{Cu}$ and $\mathrm{V}$ nuclei in $\mathrm{CuV}_{2} \mathrm{~S}_{4}$, which are used for the calculations of energy levels originating from the electric quadrupole interaction and the nuclear Zeeman effect.

\begin{tabular}{|c|c|c|c|}
\hline & ${ }^{63} \mathrm{Cu}$ & ${ }^{65} \mathrm{Cu}$ & ${ }^{51} \mathrm{~V}$ \\
\hline $\begin{array}{l}\text { Number of nuclei in } \\
\text { one molecule }\end{array}$ & \multicolumn{2}{|c|}{$1 / \mathrm{CuV}_{2} \mathrm{~S}_{4}$} & $2 / \mathrm{CuV}_{2} \mathrm{~S}_{4}$ \\
\hline $\begin{array}{l}\text { Natural abundance } \\
\text { I: Nuclear spin }\end{array}$ & $\begin{array}{l}69.09 \% \\
3 / 2\end{array}$ & $\begin{array}{l}30.91 \% \\
3 / 2\end{array}$ & $\begin{array}{l}99.76 \% \\
7 / 2\end{array}$ \\
\hline $\begin{array}{l}Q: \text { Nuclear electric } \\
\text { quadrupole moment }\end{array}$ & $\begin{array}{c}-0.16 \\
{\left[10^{-24} \mathrm{~cm}^{2}\right]}\end{array}$ & $\begin{array}{c}-0.15 \\
{\left[10^{-24} \mathrm{~cm}^{2}\right]}\end{array}$ & $\begin{array}{c}+0.2 \\
{\left[10^{-24} \mathrm{~cm}^{2}\right]}\end{array}$ \\
\hline $\begin{array}{l}\text { Nuclear magnetic moment } \\
\text { Mean moment }\end{array}$ & $2.223\left[\mu_{N}\right]$ & $2.382\left[\mu_{N}\right]$ & $5.149\left[\mu_{N}\right]$ \\
\hline$M=g_{N} \mu_{N} I=\gamma_{N} \hbar I$ & $1.15 \times 1$ & [erg/Oe $]$ & $2.60 \times 10^{-23}[\mathrm{erg} / \mathrm{Oe}]$ \\
\hline \multirow{2}{*}{$\begin{array}{l}\text { The Zeeman } \\
\text { splitting } \\
\begin{aligned} \delta=g_{N} \mu_{N} H \\
=\gamma_{N} \hbar H\end{aligned}\end{array}$} & \multicolumn{2}{|c|}{$7.66 \times 10^{-27}[\mathrm{~J}]$} & $7.43 \times 10^{-27}[\mathrm{~J}]$ \\
\hline & \multicolumn{2}{|c|}{$1.53 \times 10^{-26}[\mathrm{~J}]$} & $1.49 \times 10^{-26}[\mathrm{~J}]$ \\
\hline
\end{tabular}

E. Nuclear specific heat:

Nuclear Zeeman effect on $\mathrm{V}$ and $\mathrm{Cu}$ nuclei

Figure 10 shows a schematic energy level diagram of $\mathrm{Cu}\left(I=\frac{3}{2}\right)$ in the presence of the applied magnetic field. The axially symmetric field gradient $(\eta=0)$ is supposed.
When the angle between the principal axis of the EFG and the direction of the applied magnetic field is $\theta$, calculations for the energy levels can be made. The base wave functions $\Psi_{I, m}$ for the nuclear spin $I=\frac{3}{2}, I_{z}=m$ are $\Psi_{3 / 2,3 / 2}, \Psi_{3 / 2,1 / 2}, \Psi_{3 / 2,-1 / 2}$, and $\Psi_{3 / 2,-3 / 2}$. The eigenvalues of the Hamiltonian for the sum of quadrupolar and

TABLE III. Analysis of the nuclear specific heat of $\mathrm{CuV}_{2} \mathrm{~S}_{4}$. A quantitative calculation has been made on the basis of the coefficient for the high-temperature tail of the Schottky-type specific heat.

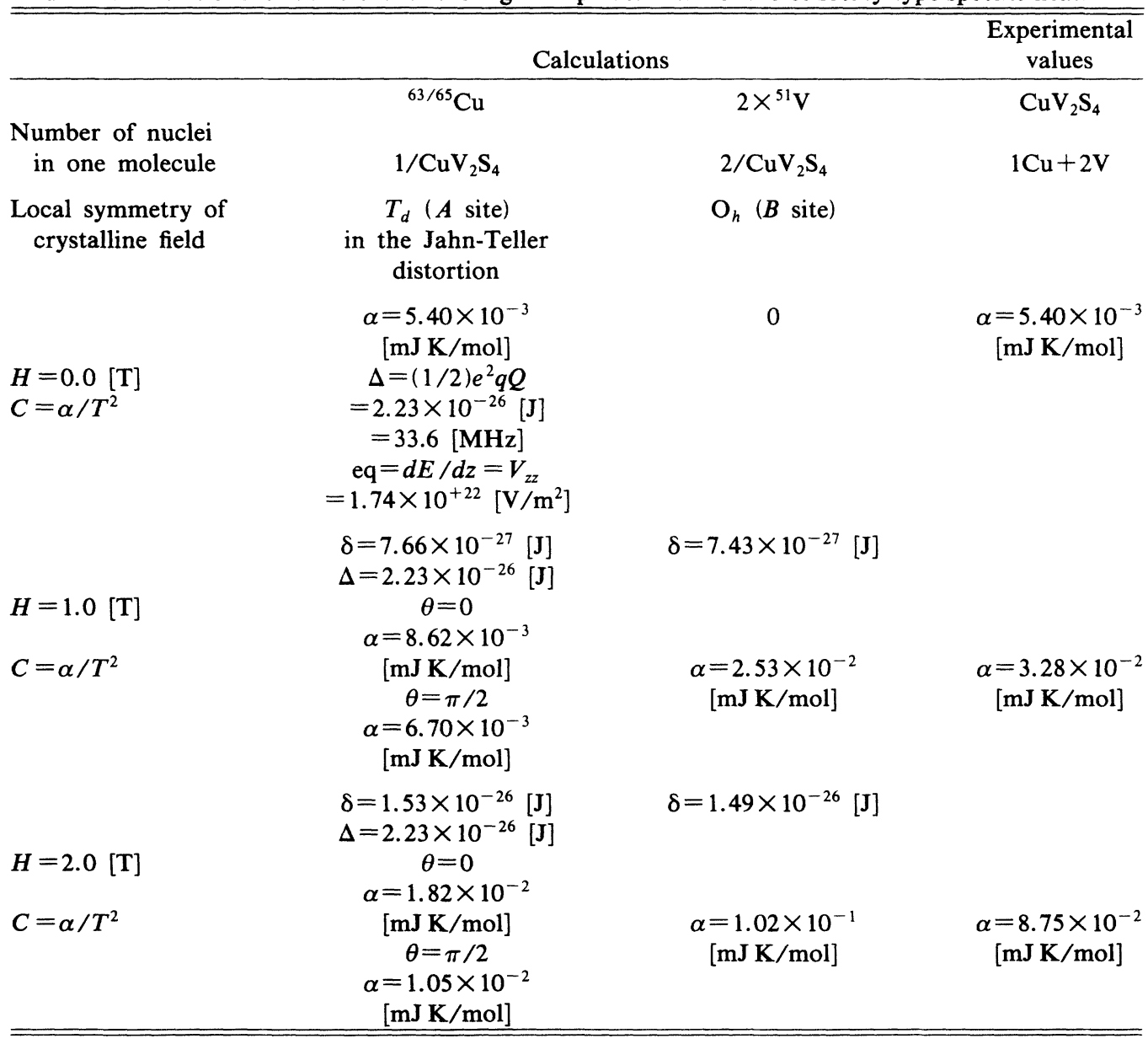


TABLE IV. Summary of the nuclear specific heat of $\mathrm{CuV}_{2} \mathrm{~S}_{4}$. In the presence of a magnetic field the contribution to the specific heat is dominated by the Zeeman effect in vanadium nuclei. In the absence of the magnetic field the specific heat comes from the electric quadrupole interaction of the copper nuclei in the electric-field gradient.

\begin{tabular}{|c|c|c|c|c|}
\hline \multirow[b]{2}{*}{$\begin{array}{l}\text { Definition of } \alpha \\
C=\alpha / T^{2}\end{array}$} & \multicolumn{4}{|c|}{ Values of $\alpha[\mathrm{mJ} \mathrm{K} / \mathrm{mol}]$} \\
\hline & ${ }^{63 / 65} \mathrm{Cu}$ & $\begin{array}{c}\text { Calculations } \\
2 \times{ }^{51} \mathrm{~V}\end{array}$ & Summation & $\begin{array}{c}\text { Experimental } \\
\text { values }\end{array}$ \\
\hline$H=0.0[\mathrm{~T}]$ & $5.40 \times 10^{-3}$ & 0 & $5.40 \times 10^{-3}$ & $5.40 \times 10^{-3}$ \\
\hline$H=1.0[\mathrm{~T}]$ & $\sim 7.7 \times 10^{-3}$ & $2.53 \times 10^{-2}$ & $3.3 \times 10^{-2}$ & $3.28 \times 10^{-2}$ \\
\hline$H=2.0[\mathrm{~T}]$ & $\sim 1.4 \times 10^{-2}$ & $1.02 \times 10^{-1}$ & $1.2 \times 10^{-1}$ & $8.75 \times 10^{-2}$ \\
\hline
\end{tabular}

Zeeman terms are given by

$$
\begin{aligned}
& \varepsilon_{1}=-\frac{\Delta}{2}-\frac{1}{2} \gamma_{N} \hbar H \sqrt{4-3 \cos ^{2} \theta}, \\
& \varepsilon_{2}=-\frac{\Delta}{2}+\frac{1}{2} \gamma_{N} \hbar H \sqrt{4-3 \cos ^{2} \theta}, \\
& \varepsilon_{3}=\frac{\Delta}{2}-\frac{3}{2} \gamma_{N} \hbar H \cos \theta, \\
& \varepsilon_{4}=\frac{\Delta}{2}+\frac{3}{2} \gamma_{N} \hbar H \cos \theta .
\end{aligned}
$$

Here we assumed that there is no mixing in the subspace between the states of $m= \pm \frac{1}{2}$ and $m= \pm \frac{3}{2}$.

The leading term in the specific heat for the hightemperature expansion for the Schottky-type specific-heat anomaly is equal to

$$
\begin{aligned}
C_{\mathrm{Sch}}(\mathrm{Cu}) & =\frac{R}{4} \times \frac{\Delta^{2}+5\left(\gamma_{N} \hbar H\right)^{2}}{k_{B}^{2}} \times \frac{1}{T^{2}}, \quad \theta=0, \\
& \equiv \alpha_{\mathrm{Cu}}(0) / T^{2}, \\
C_{\mathrm{Sch}}(\mathrm{Cu}) & =\frac{R}{4} \times \frac{\Delta^{2}+2\left(\gamma_{N} \hbar H\right)^{2}}{k_{B}^{2}} \times \frac{1}{T^{2}}, \quad \theta=\pi, \\
& \equiv \alpha_{\mathrm{Cu}}(\pi) / T^{2} .
\end{aligned}
$$

The numerical value of $\alpha$ can be obtained by using the value of $\Delta$ in Eq. (4) in Sec. III E. On the other hand, the experimental value of $\alpha$ is obtained from the specific heat shown in Figs. 8 and 9. Unfortunately, it should be noted that the experimental results of $\alpha$ can give only the averaged for the angle $\theta$. A comparison of the experimental value of $\alpha$ with the calculated value is given in Table III.

The vanadium atom has nuclear spin $I=\frac{7}{2}$, then the nuclear Zeeman splitting appears in a presence of a magnetic field. The difference between each energy level is $\gamma_{N} \hbar H$. The high-temperature specific heat is given by

$$
\begin{aligned}
C_{\mathrm{Sch}}(\mathrm{V}) & =\frac{2 R}{3} I(I+1) \frac{\left(\gamma_{N} \hbar H\right)^{2}}{k_{B}^{2}} \times \frac{1}{T^{2}}, I=7 / 2, \\
& =\frac{21 R}{2} \times \frac{\left(\gamma_{N} \hbar H\right)^{2}}{k_{B}^{2}} \times \frac{1}{T^{2}} \\
& \equiv \alpha_{V} / T^{2} .
\end{aligned}
$$

Here it should be noted that the number of vanadium atoms in one molecule is two in the formula unit. The cal- culated value of $\alpha_{\mathrm{V}}$ is estimated by using entries in Table II;

$$
\alpha_{\mathrm{V}}=2.53 \times 10^{-2}[\mathrm{~mJ} \mathrm{~K} / \mathrm{mol}], \quad H=1.0[T] .
$$

Table IV summarizes the nuclear specific heat of $\mathrm{CuV}_{2} \mathrm{~S}_{4}$. It is noted that the vanadium nuclear Zeeman effect dominates the Schottky-type specific-heat anomaly in an applied magnetic field. A comparison of the calculated values of $\alpha$ with the experimental value is in the excellent agreement.

\section{SUMMARY}

In conclusion, high-quality single crystals of $\mathrm{CuV}_{2} \mathrm{~S}_{4}$ do not reveal superconductivity in the temperature region down to $60 \mathrm{mK}$. Furthermore, previous experimental results $^{5}$ are not intrinsic to this compound. Nevertheless we cannot rule out the possibility of superconductivity below $60 \mathrm{mK}$.

The ground state of $\mathrm{Cu}^{2+}$ ions placed in $T_{d}$ symmetry experiences a Jahn-Teller distortion (local distortion) to a state of lower symmetry. This Jahn-Teller distortion leads to the existence of the EFG and then the quadrupolar interaction at $\mathrm{Cu}$ nuclei; consequently, the quadrupolar splitting contributes to the specific heat in the zero field. The electric-field gradient at $\mathrm{Cu}$ nuclei is estimated to be eq $=1.74 \times 10^{22} \mathrm{~V} / \mathrm{m}^{2}$.

Detailed analysis for the nuclear specific heat below 1.0 $K$ has been presented, and a comparison between the theoretical analysis and the experimental result is given. Below $0.2 \mathrm{~K}$, a nuclear specific heat is observed and an applied magnetic field increases the magnitude of the specific heat due to the nuclear Zeeman effects of $V$ and $\mathrm{Cu}$ nuclei and also the electric quadrupole interaction of the $\mathrm{Cu}$ nuclei. The high-temperature tail in the Schottky-type specific heat gives good agreement with theoretical calculations. The dominant contribution to the specific-heat anomaly in an applied field comes from the vanadium Zeeman effect at low temperatures.

\section{ACKNOWLEDGMENTS}

The authors would like to thank Professor Hidehiko Ishimoto and Nobuo Mori, the Institute for Solid State Physics, the University of Tokyo, for use of the facilities, and for warm encouragement. We are also grateful to our colleagues for their valuable help with the experiments and the cryogenic operation. 
${ }^{1}$ R. M. Fleming, F. J. DiSalvo, R. J. Cava, and J. V. Waszczak, Phys. Rev. B 24, 2850 (1981).

${ }^{2}$ N. Le Nagard, A. Katty, G. Collin, O. Gorochov, and A. Willig, J. Solid State Chem. 27, 267 (1979).

${ }^{3}$ T. Sekine, K. Uchinokura, H. Iimura, R. Yoshizaki, and E. Matsuura, Solid State Commun. 51, 187 (1984).

${ }^{4} J$. Mahy, D. Colaitis, D. Van Dyck, and S. Amelinckx, J. Solid State Chem. 68, 320 (1987).

${ }^{5}$ N. H. Van Maaren, G. M.Schaeffer, and F. K. Lotgering, Phys. Lett. 25A, 238 (1967).

${ }^{6}$ Y. Seki, T. Hagino, S. Takayanagi, and S. Nagata, J. Phys. Soc. Jpn. 61, 2597 (1992).
${ }^{7}$ V. D. Mahl, J. Pickardt, and B. Reuter, Z. Anorg. Allg. Chem. 491, 203 (1982).

${ }^{8}$ R. W. McCallum, D. C. Johnston, C. A. Luengo, and M. B. Maple, J. Low Temp. Phys. 25, 177 (1976).

${ }^{9}$ See, for example, J. Kanamori, J. Appl. Phys., Suppl. 31, 14S (1960).

${ }^{10}$ See, for example, T. P. Das and E. L. Hahn, in Solid State Physics: Advances in Research and Applications, edited by F. Seitz and D. Turnbull (Academic, New York, 1958), Suppl. 1, p. 1.

${ }^{11}$ E. S. R. Gopal, Specific Heats at Low Temperatures (Plenum, New York, 1966), p. 102. 


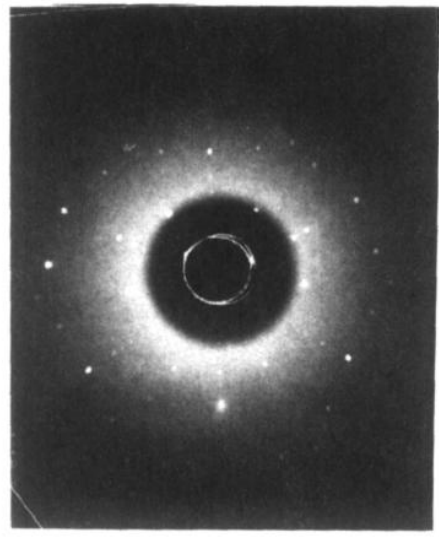

C $\mathrm{u} \mathrm{V}{ }_{2} \mathrm{~S}_{4}$

FIG. 3. Laue photograph for $\mathrm{CuV}_{2} \mathrm{~S}_{4}$ taken by back reflection from a face of octahedral-shaped single crystal. 